\title{
Recovery Orientation Among Individuals with Serious Mental IIIness
}

\author{
Aaron Fernandez ${ }^{*}$, Kit-Aun Tan, Ruziana Masiran \\ Department of Psychiatry, Faculty of Medicine and Health Sciences, Universiti Putra Malaysia, Serdang, Malaysia \\ Email address: \\ aaron@upm.edu.my (A. Fernandez) \\ ${ }^{*}$ Corresponding author
}

To cite this article:

Aaron Fernandez, Kit-Aun Tan, Ruziana Masiran. Recovery Orientation Among Individuals with Serious Mental Illness. American Journal of Applied Psychology. Vol. 6, No. 4, 2017, pp. 71-74. doi: 10.11648/j.ajap.20170604.14

Received: April 17,2017; Accepted: April 27, 2017; Published: October 18, 2017

\begin{abstract}
In the present study, we examined differences between individuals with schizophrenia and individuals with neuroses in a suburban clinical sample with respect to recovery orientation. A sample of 100 psychiatric patients from one public hospital in Selangor, Malaysia participated in this study. Participants' recovery orientation was assessed by the Recovery Assessment Scale Questionnaire. The Multivariate Analysis of Variance (MANOVA) was significant. Univariate tests further showed that there was a significant difference across two different diagnoses on reliance on others. In particular, individuals with neuroses had higher reliance on others than individuals with schizophrenia did. In an attempt to promote recovery orientation among individuals with serious mental illness, social connection and social support are domains that mental health care providers could target on.
\end{abstract}

Keywords: Recovery Orientation, Serious Mental Illness, Social Connection, Reliance on Others

\section{Introduction}

The current management paradigm of serious mental illness (SMI) has an overwhelming focus on psychiatric symptoms (e.g., hallucinations, delusions, and mood disorders). Treatments include reduction, amelioration or remission of such symptoms. The concept of recovery in mental illness has evolved from impairment model to recovery model. As opposed to recovery model, impairment model posits that recovery was a dichotomous outcome. Recovery model, however, views recovery as a process characterized by the presence of positive attributes. According to [1], positive attributes pertaining to recovery orientation among individuals with SMI include a set of positive attitudes (e.g., searching a hope of recovering). Other positive recovery attitudes include empowerment and having necessary skills to cope with symptoms and stigma [2].

One issue concerning recovery orientation is that of clinical assessment. Such assessment tools are crucial for determining recovery orientation, and for identifying effective health care management, of SMI individuals [3]. A few domains pertaining to recovery orientation derived considerably from thematic analyses. The Recovery Assessment Scale (RAS) is designed to measure recovery orientation [4] and has good psychometric properties [5]. Due to the focus of this study, reliance on others, one of the domains of the RAS, was the primary outcome. Reliance on others domain measures perception of social support and belief about the importance of social network.

Social Network refers to a system or network of interconnected persons connected by virtue of interpersonal relationships.

Social connection is a primary domain of social network [7]. Individuals with SMI have poorer social connection as compared to other clinical and non-clinical cohorts [8]. In particular, as compared to individuals with neuroses, individuals with schizophrenia reported a consistent pattern of high social isolation and low social connection [9]. Resulting from their relative lack of reciprocity in social relationships, individuals with schizophrenia have limited social connection to care providers and mental health care 
workers [10].

Social support, another domain of social network, reflects the presence of adequate social connection from significant others in time of need [11]. There are positive links between social support and recovery, and quality of life [12]. It is also documented that social support is one of the necessary criteria in determining recovery in schizophrenia [13].

\section{From Social Stigma to Self-Stigma}

The other subtle but equally detrimental effect of SMI is that of stigma. Social stigma is a mark that separates one from others and is usually associated with a host of negative discrediting characteristics ranging from beliefs, to emotions, and to behaviour [14]. Individuals with mental illness are largely aware of the stigma associated with their illness. There are two prerequisites of internalization of stigma [15]. First, identification of persons with SMI with the stigmatized group. Second, legitimation of social stigma about mental illness. Taken together, social stigma could be transformed into self-stigma via the process of internalization such as low levels of self-esteem and selfexpectation and high levels of withdrawal behaviours [16], [17] \& [14]. In turn, poor self-perception and anticipation of rejection could result in poor social connection and social support [18]. For example, individuals with selfstigma are more unlikely to seek out help or to have work and housing opportunities [19].

\section{The Present Study and Predictions}

In the present study, we examined differences in recovery orientation between individuals with schizophrenia and individuals with neuroses in a suburban clinical sample from Malaysia. Deficits in social connection may affect individuals with schizophrenia in both perceiving and receiving social support. They may have greater difficulties in procuring social support due to impaired social skills and poor social competence [20]. It is also plausible that individuals with schizophrenia are less likely than their depressed counterparts to perceive others as caring or supportive. They are also less inclined to believe in the importance of social network [9] \& [18]. Based on previous findings, we hypothesized that individuals with neuroses would exhibit a higher level of recovery orientation in terms of reliance on others as compared to individuals with schizophrenia.

\section{Methods}

\subsection{Participants}

A sample of one-hundred psychiatric patients (53 males and 47 females) was obtained from one public hospital in Selangor, Malaysia. Participants' age ranged from 19 to 91 years old $(M=46.67, S D=14.67)$. As far as diagnosis of the patients is concerned, $37 \%$ of the participants had neuroses and $63 \%$ had schizophrenia. The ethnic composition in the current sample was $44 \%$ Chinese, $38 \%$ Malay, and $18 \%$ Indian.

\subsection{Consent}

Prior to data collection, the corresponding author obtained ethical clearance from the Ethics Committee for Research Involving Human Subjects, Universiti Putra Malaysia. He also obtained permission and approval from the National Medical Research Ethics Committee. Participation was strictly voluntary. After informed consent had been sought, two medical students invited all participants to complete the questionnaires.

\subsection{Measures}

Recovery Assessment Scale [4] Participants' recovery orientation in terms of personal confidence and hope, willingness to ask for help, goal and success orientation, reliance on others, and non-domination by symptoms were assessed by the RAS. Participants provided ratings for these domains based on a 5-point Likert scale ranging from 1 (strongly disagree) to 5 (strongly agree). Internal consistency reliability estimate was .73 for the present sample.

Demographic Information. Participants also completed a demographic information sheet, including age, gender, ethnicity, marital status, educational level, and employment status.

\section{Results}

\section{Demographic Information}

Table 1. Presents a summary of demographic information for the present sample.

\begin{tabular}{ll}
\hline Variable & Statistics \\
\hline Age, $M(\mathrm{~S} D)$ & $46.67(14.67)$ \\
Gender, $n(\%)$ & 53 \\
Male & 47 \\
Female & \\
Ethnicity, $n(\%)$ & 44 \\
Chinese & 38 \\
Malay & 18 \\
Indian & \\
Marital status, $n(\%)$ & 25 \\
Single & 63 \\
Married & 2 \\
Divorced & 10 \\
Widowed & \\
Educational level, $n(\%)$ & 17 \\
Primary & 57 \\
Secondary & 9 \\
Tertiary & 17 \\
None & \\
Employment status, $n(\%)$ & 40 \\
Employed & 49 \\
Unemployed & 3 \\
Part-time & 8 \\
Self-employed &
\end{tabular}

Descriptive Statistics 
Table 2. Shows the intercorrelations, means, and standard deviations for the variables in the analysis. All correlations among study variables were statistically significant.

\begin{tabular}{llllll}
\hline Variable & $\mathbf{1}$ & $\mathbf{2}$ & $\mathbf{3}$ & $\mathbf{4}$ & $\mathbf{5}$ \\
\hline 1 PCNH & & & & & \\
$2 \mathrm{WTAFH}$ & $.51^{*}$ & & & & \\
$3 \mathrm{GNSO}$ & $.47^{*}$ & $.45^{*}$ & & & \\
$4 \mathrm{ROO}$ & $.54^{*}$ & $.51^{*}$ & $.47^{*}$ & & \\
$5 \mathrm{NDBS}$ & $.57^{*}$ & $.50^{*}$ & $.42^{*}$ & $.34^{*}$ & \\
$M$ & 24.76 & 10.69 & 18.2 & 13.78 & 10.30 \\
$S D$ & 4.38 & 2.36 & 3.73 & 2.62 & 2.62 \\
\hline
\end{tabular}

Note. $\mathrm{PCNH}=$ Personal Confidence and Hope, WTAFH $=$ Willingness to Ask for Help, GNSO = Goal and Success Orientation, ROO $=$ Reliance on Others, NDBS = No Domination by Symptoms.

$* p<.01$

\section{Multivariate Analysis}

The authors performed a one-way Multivariate Analysis of Variance (MANOVA) to examine the attitudes about recovery between individuals with neuroses and individuals with schizophrenia. The five scales serving as DVs were in agreement with constructs concerning personal confidence and hope, willingness to ask for help, goal and success orientation, reliance on others, and no domination by symptoms. A non-significant Box's M test $(p=.50)$ indicates homogeneity of covariance matrices of the dependent variable across two different psychiatric diagnoses. The MANOVA was significant, $F(5,94)=2.54, p<.05$; Wilk's $A$ $=2.54$, partial $\eta^{2}=.12$. Univariate tests showed that there was a significant difference across two different diagnoses on reliance on others, $F(1,98)=5.99, p<.01$, partial $\eta^{2}=.06$. In particular, individuals with neuroses had higher reliance on others as compared to those with schizophrenia.

\section{Discussion}

To the best of the authors' knowledge, this is the first study to assess recovery orientation using a psychiatric sample in Malaysia. The preliminary findings obtained indicate that individuals with neuroses had higher reliance on others than individuals with schizophrenia did. There are several possible reasons to explain these findings. Firstly, social isolation and withdrawal are more pronounced among individuals with schizophrenia compared to individuals with neuroses. Lack of social contact results in low opportunities to establish supportive personal relationships [11]. Secondly, in comparison to individuals with schizophrenia, self-concept, esteem and-efficacy may be higher among individuals with depression. As a result of social and self-stigma, persons with schizophrenia are more likely to identify negative stereotypes and prejudice associated with their mental illness [14], [16] $\&$ [19]. Thirdly, persons with depression are more likely to endorse a rich social network compared to persons with schizophrenia. It is possible that individuals with schizophrenia have a lack of diversity with respect to social network [9]. To this end, they are less likely to experience the benefits of having someone who can provide instrumental and emotional support.

A few limitations should be noted. First, the enumerators were not clinicians and the diagnosis was primarily based on case-notes. They were not familiar with psychiatric diagnoses and they were asked to classify anxiety and depressive disorders as neuroses. To increase data robustness, all diagnoses could have been confirmed by trained individuals using a short structured clinical interview such as the Miniinternational neuropsychiatric interview (M.I.N.I) [21]. Second, only intrinsic factors (i.e., beliefs) pertaining to recovery orientation were assessed in the present study using the RAS. Extrinsic factors (e.g., the recovery orientation of mental health practices) which are also predictive of the recovery process were not assessed. Recovery orientation with respect to mental health practices should be assessed using available outcome measures in future studies [3].

In conclusion, this study found that individuals with neuroses had higher reliance on others as compared to individuals with schizophrenia. In their attempt to promote recovery orientation among individuals with SMI, mental health care providers should be highly aware of the poorer social connections in persons with schizophrenia and should therefore evaluate, address and intervene to reduce or eliminate deficits in social connection and social support wherever possible.

\section{References}

[1] Andresen R, Oades L, Caputi P. The experience of recovery from schizophrenia: towards an empirically validated stage model. Aust N Z J Psychiatry. 2003; 37 (5): 586-94.

[2] Schrank B, Slade M. Recovery in psychiatry. Psychiatric Bull 2007; 31:321-5.

[3] Orde TC, Chamberlin J, Carpenter J, Leff HS. Measuring the promise: A compendium of recovery measures. Evaluation Center@HRSI; 2005.

[4] Giffort D, Schmook A, Woody C, Vollendorf C, Gervain M. Construction of a scale to measure consumer recovery. Springfield, IL: Illinois Office of Mental Health. 1995

[5] Corrigan PW, Salzer M, Ralph RO, Sangster Y, Keck L. Examining the factor structure of the recovery assessment scale. Schizophr Bull. 2004; 30 (4): 1035-41.

[6] Salzer MS, Brusilovskiy E. Advancing recovery science: reliability and validity properties of the Recovery Assessment Scale. Psychiatr Serv. 2014; 65 (4): 442-53.

[7] Stanghellini G, Ballerini M. Dis-sociality: the phenomenological approach to social dysfunction in schizophrenia. World Psychiatry. 2002; 1 (2): 102-6.

[8] Tsai J, Desai RA, Rosenheck RA. Social integration of people with severe mental illness: relationships between symptom severity, professional assistance, and natural support. J Behav Health Serv Res. 2012; 39 (2): 144-57.

[9] Goldberg RW, Rollins AL, Lehman AF. Social network correlates among people with psychiatric disabilities. Psychiatr Rehabil J. 2003; 26 (4): 393-402. 
[10] Macdonald EM, Hayes RL, Baglioni AJ, Jr. The quantity and quality of the social networks of young people with early psychosis compared with closely matched controls. Schizophr Res. 2000; 46 (1): 25-30.

[11] Berkman LF, Glass T, Brissette I, Seeman TE. From social integration to health: Durkheim in the new millennium. Soc Sci Med. 2000; 51 (6): 843-57.

[12] Corrigan PW, Phelan SM. Social support and recovery in people with serious mental illnesses. Community mental health journal. 2004 Dec 1; 40 (6): 513-23.

[13] Liberman RP, Kopelowicz A, Ventura J, Gutkind D. Operational criteria and factors related to recovery from schizophrenia. International Review of Psychiatry. 2002 Jan 1; 14 (4): 256-72.

[14] Rusch N, Angermeyer MC, Corrigan PW. Mental illness stigma: concepts, consequences, and initiatives to reduce stigma. Eur Psychiatry. 2005; 20 (8): 529-39.

[15] Corrigan PW, Watson AC. The paradox of self-stigma and mental illness. Clinical Psychology: Science and Practice. 2002 Mar 1; 9 (1): 35-53.
[16] Corrigan PW. The impact of stigma on severe mental illness. Cognitive and behavioral practice. 1999 Feb 28; 5 (2): 201-22.

[17] Drapalski AL, Lucksted A, Perrin PB, Aakre JM, Brown CH, DeForge BR, et al. A model of internalized stigma and its effects on people with mental illness. Psychiatr Serv. 2013; 64 (3): 264-9.

[18] Anthony WA. Recovery from mental illness: The guiding vision of the mental health service system in the 1990s. Psychosocial rehabilitation journal. 1993 Apr; 16 (4): 11.

[19] Ritsher JB, Otilingam PG, Grajales M. Internalized stigma of mental illness: psychometric properties of a new measure. Psychiatry Res. 2003; 121 (1): 31-49.

[20] Stanghellini G, Ballerini M. Dis-sociality: the phenomenological approach to social dysfunction in schizophrenia. World Psychiatry. 2002; 1 (2): 102-6.

[21] Sheehan DV, Lecrubier Y, Sheehan KH, Amorim P, Janavs J, Weiller E, et al. The Mini-International Neuropsychiatric Interview (M.I.N.I.): the development and validation of a structured diagnostic psychiatric interview for DSM-IV and ICD-10. J Clin Psychiatry. 1998; 59 Suppl 20: 22-33; quiz 457. 